PART 2.

\title{
Black Hole Transient Sources
}




\title{
High Energy Continuum Spectra from X-Ray Binaries
}

\author{
S.N. Zhang
}

USRA/Marshall Space Flight Center, ES-84, Huntsville, AL 35812

\begin{abstract}
A variety of high energy $(>1 \mathrm{keV})$ spectra have been observed in recent years from Black Hole (BH) and Neutron Star (NS) $\mathrm{X}$-ray Binaries (XB). Some common physical components exist between BHXBs and NSXBs, resulting in some high energy spectral features. A common component between a BHXB and a weakly magnetized NSXB is the inner accretion disk region extending very close to the surface (for a NS) or the horizon (for a $\mathrm{BH}$ ). The inner disk radiation can be described by a multi-color blackbody (MCB) spectral model. The surface radiation of the NS can be approximated by a Single Color Blackbody (SCB) spectrum. For a strongly magnetized NSXB, the high energy emission is from its magnetosphere, characterised by a thermal bremsstrahlung (TB) spectrum. In both BHXBs and weakly magnetized NSXBs, a hot electron cloud may exist, producing the hard X-ray power law (photon index -1.5 to -2.0$)$ with thermal cutoff $(50-200 \mathrm{keV})$. It has been recently proposed that a converging flow may be formed near the horizon of a $\mathrm{BH}$, producing a softer power law (photon index about -2.5) without cutoff up to several hundred $\mathrm{keV}$. Based on these concepts we also discuss possible ways to distinguish between $\mathrm{BH}$ and NS XBs. Finally we discuss briefly spectral state transitions in both BH and NS XBs.
\end{abstract}

\section{Introduction}

Following the launches of several high energy astrophysical satellites in the last decade, for example, EXOSAT, TTM and HETE aboard Mir Space Station, ROSAT, Ginga SIGMA/GRANAT, CGRO, ASCA, SAX and RXTE, our knowledge of high energy spectra from XB sources has increased enormously. In this review paper, we shall focus on the high energy spectra of these binary systems whose primary electromagnetic energy output is above $1 \mathrm{keV}$. We will, however, not discuss narrow or broad spectral line features.

\section{Spectral formations in X-ray binaries}

In a binary system involving a compact object (a NS or a $\mathrm{BH}$ ) and a more ordinary star (called the companion star), matter can be transferred, either through Roche lobe overflow or via a stellar wind, from the companion to the compact object. In order for the transferred or accreted matter to reach the compact object, angular momentum loss has to occur, presumably via viscous mechanisms. Thus an accretion disk may be formed and the gravitational energy loss via the viscous loss will be radiated in the form of electromagnetic waves. The energy of 
the radiated photons will become higher when the accreted material gets closer to the central object. In the standard Shakura-Sunyaev (1973) model, a geometrically thin and optically thick accretion disk is formed. The local radiation can be approximated by a blackbody spectrum. The overall spectrum is thus the integration of the local (effective) blackbody emission from the inner disk region to the outer disk edge (see e.g., Mitsuda et al. 1984, 1989). Such a spectrum model is called the MCB model. The majority of the total luminosity is radiated between the inner disk edge (a few $R_{s}$, the Schwarzschild radius) to a radius of the order of $100 R_{s}$. The inner edge temperature for a stellar mass $\mathrm{BH}$ is usually around $1 \mathrm{keV}$. A MCB spectrum is shown in figure 1 together with other spectral models to be discussed in this section.

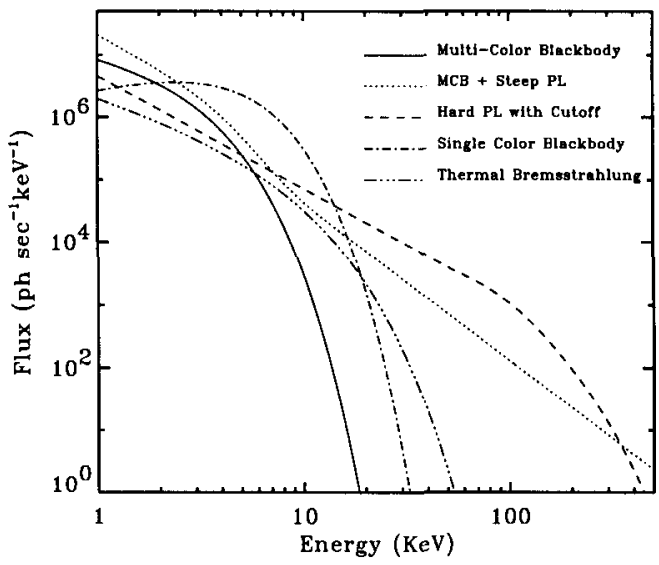

Figure 1: Characteristic high energy spectral shapes formed in X-ray binaries. The relative normalizations are arbitrary, in order to show all of them clearly. The possible origin of each spectral component: inner accretion disk region $\Rightarrow$ multi-color blackbody, neutron star surface $\Rightarrow$ single color blackbody, neutron star magnetosphere $\Rightarrow$ thermal bremsstrahlung spectrum, hot electron cloud $\Rightarrow$ hard power law with cutoff, and converging inflow $\Rightarrow$ steep power law associated with a bright MCB.

When the central compact object is a NS, the remaining gravitational energy will be released when the accreted matter finally reaches the NS. In the case of a weakly magnetized NS ( $B \leq 10^{8}$ gauss), usually found in atoll sources, the matter can reach the NS surface directly and the radiated spectrum can be approximated by the SCB model (1-2 keV) (Mitsuda et al. 1984), although the boundary layer of the NS is quite complicated. The measured temperature is thus the effective temperature of the NS surface. In the $1-10 \mathrm{keV}$ region, the MCB spectrum is considerably softer than the SCB spectrum. This is why the MCB spectrum is usually called an Ultra-Soft (US) spectrum.

In case of a strongly magnetized NS ( $B \geq 10^{10}$ gauss), usually found in $\mathrm{X}$ ray pulsars, the accreted matter cannot reach the NS surface directly and is funneled onto its polar cap region by the strong magnetic field. A hot plasma 
(5-20 keV) is thus formed and the continuum emission can be described by the TB, characterised by a hard power law (photon index -2.0 to -1.0 ) with an exponential cutoff below $20 \mathrm{keV}$ (White, Swank \& Holt 1983). For a mildly magnetized NS $\left(10^{9}<B<10^{10}\right.$ gauss), usually found in Z-sources, both the SCB and the TB components may be present, so that the overall spectrum becomes quite soft compared with X-ray pulsars.

In some systems Roche lobe overflow cannot occur. Significant wind accretion will take place if the companion can produce strong winds. Such systems exist with either a NS (e.g., Vela X-1) or a BH (e.g., Cyg X-1) as the compact object. As well as forming an accretion disk, the wind accretion in a $\mathrm{BH}$ system may cause some interesting consequences. As suggested by Shapiro and Lightman (1976), an asymmetric shock front (bow shock) will be formed with a temperature of the order of $10^{9} \mathrm{~K}$, when the $\mathrm{BH}$ accretes directly from the stellar wind of its High Mass (HM) companion as in Cyg X-1. It is reasonable to expect that the thermal radiation from the accretion disk, if formed, will be inverse Compton scattered to higher energy photons (Zhang et al. 1997a) by the hot electrons in the bow shock (the electrons are heated up by collisions with protons). As calculated by Sunyaev and Titarchuk (1980) the spectrum will be characterized by a power law with a high energy cutoff, whose energy is proportional to the mean electron temperature in the shock. This mechanism may naturally explain the observed hard state spectrum of Cyg X-1 (photon index -1.5 to -2.0 with cutoff between $50-200 \mathrm{keV}$ ). This is the so-called TC process. In figure 2, we show an illustrative model of Cygnus X-1 (Zhang et al. 1997a).

Even in Low Mass (LM) XBs, some accretion disk models also suggest the existence of a hot cloud surrounding the central compact object. Thus the TC process may also work here. Several models are invoked to explain the formation of this cloud. The most recent ones are the two-phase model of Haardt and Marashi (1993), advection-dominated model of Narayan (1996) and the postshock model of Chakrabarti and Titarchuk (1995). Although this issue is still controversial, this hot cloud seems to exist in both BH and NS XBs and Seyfert galaxies as well, as inferred from the characteristic TC spectra.

When the supply of the low energy photons is over-abundant, this TC process can cool down the cloud and eventually destroy it. In the case of a NS $\mathrm{XB}$, the power law component will decrease and finally disappear. For a $\mathrm{BH} \mathrm{XB}$, the situation is more interesting. Because of the strong gravitational field and the absorptive horizon of the $\mathrm{BH}$, the low temperature electrons will move towards the $\mathrm{BH}$ relativistically to form a converging flow (Chakrabarti and Titarchuk 1995). The relativistic electrons will up-scatter the low energy photons, either trapped in the flow when it sweeps through the surface of the accretion disk or simply intercepted by the flow, to form a power law. We call this radiation mechanism Bulk Motion Comptonization (BMC) process. Calculations made by Chakrabarti and Titarchuk (1995) have shown that the BMC power law is steeper (photon index around -2.5) than the TC power law formed from the hot Comptonization cloud (photon index -1.5 to -2.0 ). 


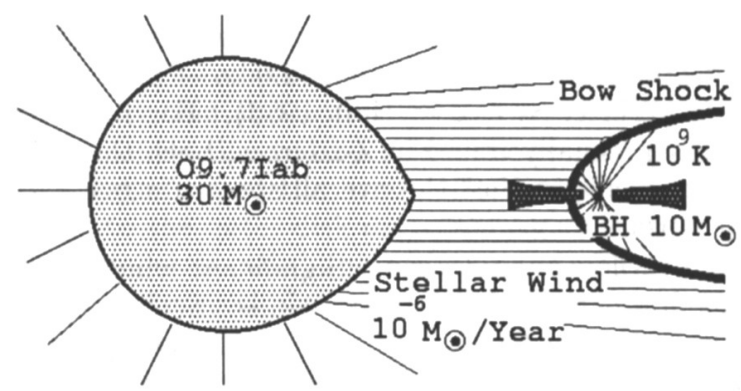

Figure 2: Illustrative Model of Cygnus X-1. The Companion is a supergiant 09.7 Iab with a mass of about 30 $\mathrm{M}_{\odot}$. The bow shock is formed when some of the low angular momentum component of the stellar wind $\left(\sim 10^{-6}\right.$ $\mathrm{M}_{\odot}$ per year) from the companion is captured by the $\sim 10$ $\mathrm{M}_{\odot}$ black hole. The accretion disk is formed out of the high angular momentum component of the wind. The blackbody-like thermal emission is produced from the accretion disk. Some of the low energy photons are inverseCompton scattered to higher energies by the hot electrons in the bow shock, forming the observed hard $X$-ray power law with a cutoff (the cutoff energy is proportional to the temperature of the bow shock, which is $\sim 10^{9} \mathrm{~K}$, but may vary depending upon the mass flow rates of the direct wind capture and the optically thick disk).

\section{Distinguishing between neutron star and black hole X-ray binaries}

As a matter of fact, up to the present time, the only reliable way of determining if a binary system contains a $\mathrm{BH}$ is by measuring the system parameters. If one can estimate the mass of the compact object, it will be considered to be BH if its mass is $>3 \mathrm{M}_{\odot}$, currently the best known theoretical and observational upper mass limit of a NS. Several systems have been identified in this way. However, this method is quite restrictive in principally two aspects. One is that the masses of the compact objects of the majority of the BH candidates (see below) cannot be reliably measured due to various observational constraints. The other is that this method in principle remains ambiguous for those with mass limits $<3 \mathrm{M}_{\odot}$. Therefore we have to look for alternative methods of selecting BHBs.

By comparing the common characteristics of the high energy emission from these dynamically determined BH systems, it has been hoped that some "signatures" of BHs may be found when accurate dynamical estimates are not available. Such "signatures" include ultra-soft/ultra-hard energy spectra, rapid variability, QPOs etc. All such features have, however, been observed from NS systems, especially those with a weak magnetic field. This is, perhaps, not surprising since most of these phenomena are associated with properties of the accretion disk common to both types of systems. It has been recently suggested that perhaps luminous hard X-ray emission or a combination of an ultrasoft component and a hard X-ray power-law are more indicative of the existence of 
a black hole (van der Klis 1994; van der Klis and van Paradijs 1993; White 1993; Tanaka \& Lewin 1995; Zhang et al. 1996a; 1996b; Barret, McClintoch \& Grindlay 1996).

In this section we try to understand how to distinguish between NS and $\mathrm{BH} \mathrm{XBs}$, based on the similarities and differences in the formation process of high energy spectra of the systems we discussed in the previous section. We will first identify some signatures of NSXBs and then discuss how a BHXB might be identified in the absence of any signature of NSXBs.

\subsection{Signatures of neutron star X-ray binaries}

The high energy spectra of magnetized NSXBs can be relatively easily distinguished from those of BHXBs. The TB spectral shape with a cutoff energy below $20 \mathrm{keV}$ is a strong indication of the existence of the NS magenetosphere and, in fact, has never been detected from a BHXB. The 1-2 keV SCB component, if unambiguously identified, is a strong indication of the detection of the neutron star surface radiation. This component has also never been detected from a $\mathrm{BHXB}$. Thus both characteristic spectral components, together with pulsations and type I X-ray bursts, can be used as signatures of NSXBs. In the absence of any one of the above signatures, a source may be considered a BHC. We now discuss how to further identify those BHXBs from the list of $\mathrm{BHCs}$, such as that given in the review by Tanaka and Lewin (1995).

\subsection{Indicators of a black hole X-ray binaries}

One common high energy radiating component between a $\mathrm{BH}$ and a weakly magnetized NS XB (or simply 'NSXB' for the rest of this section since the strongly magnetized NSXBs can be easily identified as discussed above), is the inner disk region very close to the compact object, which produces the MCB spectrum. Due to the NS SCB emission, the overall spectrum of such a NSXB may be harder than that of a BHXB. However, when the mass accretion rate is low so that the NS surface temperature is also low, the NSXB spectrum may appear indistinguishable from that of a BHXB. Thus a luminous $\left(>10^{37} \mathrm{erg} / \mathrm{s}\right)$ $M C B$ component is a strong indication of the existence of a $B H$ in a XB.

Using the $\mathrm{MCB}$ model, in principal one can determine the temperature and radius of the inner disk edge. For a NSXB, the inner disk edge should be very close to the NS surface, i.e., about $10 \mathrm{~km}$. For a BHXB, the inner disk radius can be much larger. Therefore an inner disk radius significantly larger than 10 $\mathrm{km}$, for example, greater than $30 \mathrm{~km}$, is another indication of the existence of $a B H$. This is why dynamically determined BHXBs all appear to have much larger inner disk radii than that of NSXBs (Ebisawa et al. 1991). However, for a maximally rotating Kerr BH with a mass comparable to the NS mass, its inner disk radius will not be very different from that of a NSXB. So a small inner disk radius is not always associated with a NSXB.

Another common high energy radiation component between a BHXB and a weakly magetised NSXB is a hot electron cloud, producing the observed TC power law with cutoff between 50 and $200 \mathrm{keV}$. This cloud is different from the extended corona in many of the so-called 'dipper' sources (The coronae in the 'dipper' sources have much lower temperatures and extend well above the plane of the disk, as inferred from the significant residual fluxes of the high inclination 
systems during the accretion disk eclipse by the companion.). Although such TC spectra are quite similar between NS and BH XBs, there are, however, still some differences. One is that the spectral break energy in a BHXB is frequently observed to be $>100 \mathrm{keV}$, while for NSXBs the highest observed spectral break energy is only $65 \mathrm{keV}$ (Zhang et al. 1996a). Perhaps this is because there are two soft photon sources in a NSXB (one is the disk emission and the other is the NS surface emission), in contrast to the single soft photon source in a BHXB (the disk emission). Therefore the electron temperature in a NSXB is usually lower than that in a BHXB. The same argument can be applied to the fact that the hard X-ray luminosity can be significantly higher in a BHXB. Therefore the detection of a combination of a high energy cutoff $(>100 \mathrm{keV})$ and a bright hard $X$-ray luminosity (>10 $37 \mathrm{erg} / \mathrm{s}$ above $10 \mathrm{keV}$ ) indicates strongly the existence of a $B H$ in the system.

The hard X-ray power law emission will disappear in a NSXB when the disk accretion rate is so high that the hot electron cloud is cooled down and consequently the TC mechanism can no longer effectively produce hard X-ray photons. In a BHXB, a steeper power law (SPL) should be formed in the converging flow by the BMC mechanism (Chakrabarti \& Titarchuk 1995). This emission mechanism is related to the lack of a solid surface of a $\mathrm{BH}$, and therefore may provide a signature of the $\mathrm{BH}$. The question is whether a similar high energy spectrum can be also formed from other emission mechanisms not related to the horizon of a $\mathrm{BH}$. Although hypothetically many high energy radiation mechanisms can form such a power law energy spectrum in this energy range $(10-500 \mathrm{keV})$, the conditions when such a power law is detected restricts the possibilities severely. Almost in all cases the SPL (without cutoff up to 300-500 $\mathrm{keV}$ ) is accompanied with a bright MCB component observed from a BHXB.

Synchrotron radiation is an unlikely mechanism, since the bright MCB component tends to cool down the assumed high energy electrons by inverse Compton scattering, thus preventing non-thermal radiation up to several hundred $\mathrm{keV}$. It has been widely considered that the TC process with a cooler electron cloud as the result of the more effective cooling by the higher soft X-ray flux, may be a good candidate for producing the SPL. There are, however, several problems with this picture. First, the lower electron temperature should result in a lower spectral break energy when the SPL is observed, contrary to the detected unbroken power law up to at least $300-500 \mathrm{keV}$. The other problem is the near constancy of the SPL index when both the MCB and the SPL luminosity change significantly as observed from GS1124-68 in the soft state (Ebisawa et al. 1994). Other radiation mechanisms, involving relativistic outflows or jets, are also unlikely to work, since not all BHXBs are observed with jets or signficant radio emission, or such SPL detection is not always associated with jet ejection events (Zhang et al. 1996b). Moreover, all the above mentioned mechanisms should also operate in a NSXB; but such SPL/MCB have never been detected from any confirmed NSXB. We therefore conclude that such unbroken SPL (up to $300-500 \mathrm{keV})$ associated with a luminous $M C B$ component $\left(\sim 10^{37} \mathrm{erg} / \mathrm{s}\right)$ is mostly likely produced in the converging flow near the $B H$ horizon and may be used as a distinctive signature of $B H X B s$.

In summary, there appears to be several strong suggestive indicators of the existence of a $\mathrm{BH}$ in a XB. These are: a) a luminous $\left(\sim 10^{37} \mathrm{erg} / \mathrm{s}\right) \mathrm{MCB}$ 
spectrum without the contamination of a SCB component; b) a bright $\left(\sim 10^{37}\right.$ $\mathrm{erg} / \mathrm{s}$ ) hard X-ray power law (photon index: -1.5 to -2.0 ) with high energy cutoff $(>100 \mathrm{keV})$; and c) a SPL (photon index $\sim-2.5$ ) without cutoff up to $300-500$ $\mathrm{keV}$ associated with a luminous MCB component $\left(\sim 10^{37} \mathrm{erg} / \mathrm{s}\right)$. The first two indicators are related to the lack of surface radiation from the $\mathrm{BH}$, in contrast to a NS, while last one seems to be due to the horizon of the $\mathrm{BH}$ and thus is a more secured signature of a $\mathrm{BH}$. We do not include the large inner disk radius as a $\mathrm{BH}$ indicator here, since this parameter depends upon the details of the rather complicated spectral model which is not well understood yet.

\subsection{Applications of black hole $\mathrm{X}$-ray binary indicators}

As a matter of fact, none of the known NSXBs, including all known pulsars, Z-sources and type I X-ray bursters, have been observed with one of the three high energy spectral types, with the only possible exception of Terzan-2, a type I X-ray burster. During one observation with SIGMA/GRANAT, a hard power law up to about $200 \mathrm{keV}$ was detected with a luminosity exceeding $\sim 5 \times 10^{37}$ $\mathrm{erg} / \mathrm{s}$ (Barret et al. 1992). It is, however, not clear if the emission originated from Terzan-2, or another high energy transient from that direction or within the same globular cluster. At all other occasions when it was detectable in the hard X-ray band above $20 \mathrm{keV}$, a much lower luminosity was seen.

All the dynamically confirmd BHXBs, i.e., with the compact object mass greater than $3 \mathrm{M}_{\odot}$, have displayed at least one of the three indicators. These systems are Cygnus X-1 (indicator: b and c), 0620-00 (a, c), LMC X-3 (a, c), LMC X-1 (a, c), GS 1124-683 (a, b, c), GS 2000+25 (a, c), GS 2023+33 (b), GRO J1655-40 (a, c). Here we also further examine all those remaining BHCs listed by Tanaka and Lewin (1995). These systems should also contain a $\mathrm{BH}$ according to the three indicators: GX 339-4 (a, b, c), 1354-645 (c), 1543475 (a, b), 1630-472 (a, b, c), 1705-250 (a, c), 1740.7-2942 (b), 1741-322 (c), GRS $1915+105$ (a) and 1758-258 (b). The most recently discovered transient GRS 1739-278 also belongs to this class since the indicator (c) was detected from it (Vargas et al. 1996).

\section{Spectral state transitions in X-ray binaries}

We briefly summarise the spectral state transitions in $\mathrm{BH}$ and NS XBs, aiming at understanding the dynamics in XBs. Here we call these states 'hard' or 'soft', instead of 'low' or 'high' used by some others, since we will show below that the bolometric luminosity, presumably proportional to the overall mass transfer rate, actually changes insignificantly or at least the hard state bolometric luminosity is not necessarily always lower than that in the soft state.

\subsection{Transitions between the hard and soft states in Cyg X-1}

The most recent soft state of Cyg X-1 has been extensively observed by several high energy instruments from aboard CGRO, RXTE, ASCA and SAX satellites. Here we only show some results from the broad band monitoring achieved with ASM/RXTE (1.3-12 keV) and BATSE/CGRO (20-200 keV) (Zhang et al. $1997 \mathrm{~b})$. The main results are: a) during the whole period the soft X-ray fluxes 
are anti-correlated with the hard X-ray fluxes; b) the $1.3-200 \mathrm{keV}$ luminosity (and the bolometric luminosity after the color correction, see Zhang et al. 1997c for details) does not change significantly, and is not correlated with the state transition; c) the transition time of the lowest energy band (1.3-3.0), whose flux is dominated by the MCB component, is much shorter than that of the hard X-ray flux; d) flares with durations of around 10-30 days are seen in all energy bands, superposed on top of the state transition; and e) the soft X-ray flares lag behind the hard X-ray flares by 10-20 days.

The near constant luminosity during the state transitions defies most of the current models of high energy radiation in BHXBs. All these models require a significant luminosity increase in the soft state, although some fine tuning of some of these models may be able to remove this requirement. For example, in the Chakarabarti and Titarchuk (1995) model, at a certain mass accretion rate the re-distribution of the mass flow rates between the Keplerian and subKeplerian mass accretion components may also produce a constant luminosity during a state transition (Titarchuk 1996), instead of requiring a total mass acrretion rate increase. However, the other results, especially the longer hard X-ray flux transition time and the lag between the soft and hard flares cannot be naturally accounted for within these current models.

\subsection{State transitions in GS 1124-683 = Nova Muscae}

There appears to be several spectral state transitions observed from the LM BHXB GS 1124-683 during its 1991 outburst (see Ebisawa et al. for details of this outburst observed with Ginga). Here we just briefly mention two behaviors. The first one is during the initial outbursting phase, when the power law component reached its peak flux about 3 days earlier than the MCB component. This behavior is not really a spectral state transition, but instead reflected a lag of the MCB component increase behind the power law component increase, in responding perhaps to the mass accretion rate increase. It is interesting to note that when the soft component reached its peak, the hard component also experienced a dip, without significant changes of the spectral shape of each component. The second one is a real spectral state transition between April and July, when the hard component reached a secondary maximum and the soft component continued to decrease. The significant features of this state transition are the low luminosity level ( $\sim 1 \%$ of the peak total luminosity) when the transition happened and the much harder power law (photon index changed from between -2.2 and -2.7 to $\sim-1.6$ ) during this secondary outburst.

\subsection{Soft to hard state transition in $4 \mathrm{U}$ 1608-522}

In figure 3 we show the spectral transition of the LM NSXB 4U 1608-522 when the energy spectrum changed from a typical soft state spectrum (MCB plus a $\mathrm{SCB}$ ) to a hard state (power-law dominated) spectrum when the total luminosity decreased from about $5 \times 10^{37}$ to about $7 \times 10^{36} \mathrm{erg} / \mathrm{sec}$. A transition happened at $10^{37} \mathrm{erg} / \mathrm{sec}$. At this point the $\mathrm{MCB}$ component $\left(\mathrm{F}_{\mathrm{BD}}\right)$ decreased by nearly a factor of 3 , the hard component $\left(\mathrm{FC}_{\mathrm{C}}\right)$ (believed due to $\mathrm{TC}$ in an optically thin region) increased by about a factor of 2 and the $\mathrm{SCB}\left(\mathrm{F}_{\mathrm{NS}}\right)$ remained almost constant or increased slightly. The total X-ray luminosity tracks the total mass accretion in the system since the majority of the gravitational energy is released 
in the X-ray energy band. Therefore during this transition the change in total mass accretion rate is very small. This is also indicated by the near constant $\mathrm{SCB}$ component during the spectral state transition. The sudden decrease of the MCB component indicates the mass accretion rate through the inner edge of the disk decreased by about a factor of 3 . This material must be channeled to the region producing the observered hard X-ray component which increased simultaneously.

\subsection{Possible Interpretations}

A successful model has to account for several major phenomena. These are: a) the soft X-ray component lags behind the hard component during the flares or onsets of outbursts (hard precursors are common among soft X-rat transients or X-ray novae); b) no significant luminosity changes occur during state transitions (the spectral state transitions are usually shown as soft/hard component anticorrelations. After removing the trend of long term light curve exponential decay during the GS 1124-68 spectral state transition, the total luminosity changes are also not significant.); and c) the state transition time of the soft component is much shorter than that of the hard component (probably only observed from Cyg X-1, since no other sources have been monitored continuously over the whole transition period in a broad energy band). Since all three phenomena are observed from Cyg X-1, we will examine this system first.

In the model we show in figure 2 for Cyg X-1, phenomenon- $a$ can be explained as due to the time difference between the disk mass transfer and the direct wind capture by the $\mathrm{BH}$. When the mass flow rate carried by the stellar wind increases suddenly, the flow will reach the bow shock promptly, resulting in an increased hard X-ray flux, due to the increased Comptonization factor, although the supply of the seed photons remains the same. The mass accretion component transported through the optically thick disk will arrive later at the inner disk region, thus producing the delayed soft X-ray flux. The delay time of around 15 days constrains the optically thick Keplerian disk to fill only $1-10 \%$ of the Roche lobe of the system (Zhang et al. 1997a). Phenomenon- $b$ suggests that the the total mass accretion rate remains nearly a constant while both soft and hard X-ray fluxes change significantly. It is therefore reasonable to assume what really happened was the re-distribution of mass flow between the direct wind capture component and the optically thick disk component. Phenomenon$c$ is explained as due to the mass flow exchange between the two mass accretion components when an instability is transported between the outer and inner disk regions (see Zhang et al. 1997a for details).

Although no significant wind accretion exists in LMXBs, many models will require the existence of an optically thin, sub-Keplerian region, responsible for the hard X-ray production, although the origin and the nature of this region distinguishes these models. In this picture, the optically thick disk nearly fills the Roche lobe, since it is formed out of Roche lobe overflow, and the optically thin region surrounds the inner disk region nearly spherically. The time lag is then the same time difference, but starting from the boundary of the optically thin region since its radius is expected to be smaller than the outer radius of the optically thick disk. Thus the time lag measures the size of the optically thin region in a $L M X B$. In GS 1124-68, the lag time of $\sim 3$ days suggests the 


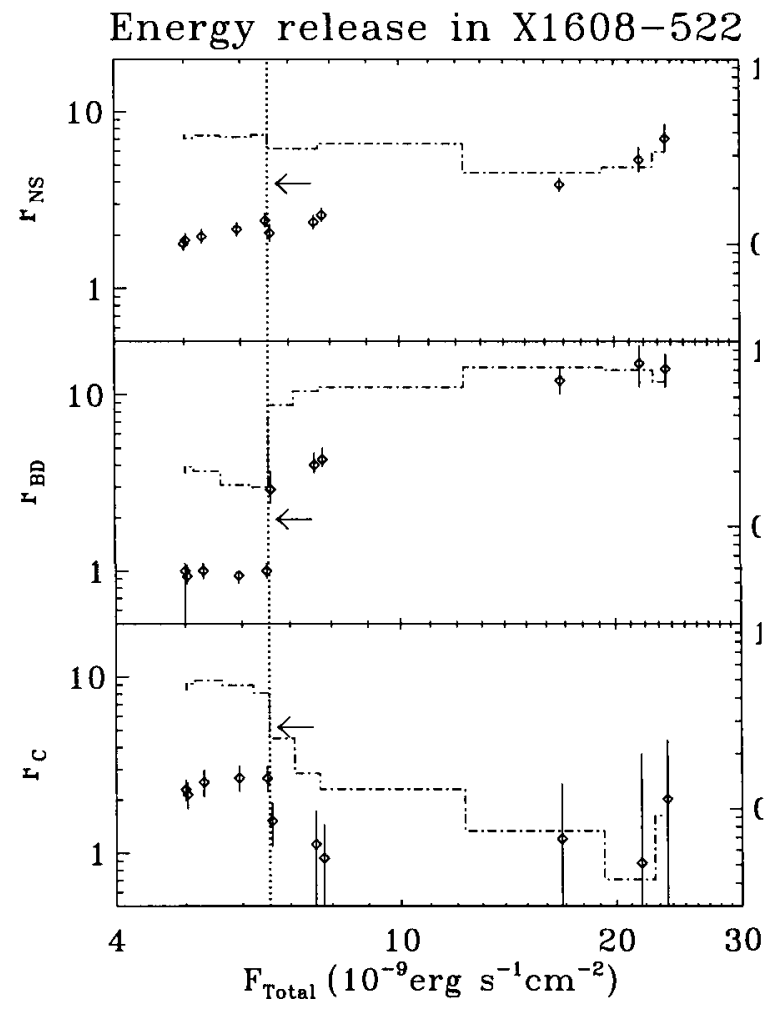

Figure 3: Energy spectral state transition observed from 4U 1608-522 with Tenma. $\mathrm{F}_{\text {total }}$ is the total detected energy flux, which can be further divided into three components. $F_{N S}$ is the SCB emission from the NS surface, $F_{B D}$ is the MCB Component from the inner region of the optically thick disk and $\mathrm{F}_{\mathrm{C}}$ is the energy flux added by a Comptonization process (all in unit of $10^{-9} \mathrm{erg} \mathrm{s}^{-1} \mathrm{~cm}^{-2}$ ). Diamonds denotes the energy fluxes and the dot-dashed lines are the percentages of these three components within the total observed energy flux. A transition happened when the total luminosity was about $6.5 \times 10^{-9} \mathrm{erg} \mathrm{s}^{-1} \mathrm{~cm}^{-2}$, corresponding to $\sim 10^{37} \mathrm{erg} \mathrm{s}^{-1}$ for at $\sim 3.6 \mathrm{kpc}$. While the total luminosity did not change significantly, the gravitational energy release was mostly transferred from the optically thick disk to the Comptonization region, possibly the optically thin mass accretion region (Data from Tenma observations described by Mitsuda et al. 1989).

size of this region is between $10^{9}$ to $10^{10} \mathrm{~cm}$, i.e, about $10-100$ times larger than the inner disk radius for a stellar mass BH. During the Cen X-4 outburst in 1979 (Bouchacourt et al. 1984), a similar lag was also found, indicating a similar geometry in both systems. A significant difference between Cyg X-1 and these LMXBs, in terms of spectral state transitions, is that the hard state is the 
persistent and dominating state and occurs at a much higher luminosity level in Cyg X-1 than the LMXBs. This is, perhaps due to the relatively higher mass flow rates in the optically thin region originated from the direct stellar wind capture in our model of Cyg X-1, than that in LMXBs, where the optically thin region is probably formed out of the optically thick disk and is much less effective when the disk mass accretion rate is very high.

\section{Summary}

It appears that a characteristic high energy spectral component is formed from each component of a NS or BH XB. Based on the similarities and differences of the spectral formation process in NS and $\mathrm{BH}$ XBs, we discussed possible ways to distinguish between NS and BH XBs. Spectral state transitions in XBs are discussed for three systems, HMBH XB Cyg X-1, LMBH XB GS 1124-68 and LMNS XB 4U 1608-522. Geometries of these systems can be constrained from the observed spectral transition characteristics.

Acknowledgments. We benefitted greatly from the many stimulating discussions between the BATSE team members, especially Alan Harmon, Bill Paciesas and Craig Robinson at Marshall Space Flight Center and with Drs. Ken Ebisawa, Lev Titarchuk, Sandip Chakrabarti, Wei Cui, Wan Chen, Jan van Paradijs, Didier Barret, Ron Remillard, Bob Hjellming and Ramesh Narayan.

\section{References}

Barret, D.et al. 1991, ApJ 379, 21

Barret, D., McClintock, J.E., Grindlay, J.E., 1996, ApJ372, in press

Bouchacourt, P. et al. 1984, ApJL 285, 67-70

Chakrabarti, S.K. \& Titarchuk, L. G. 1995, ApJ, 455, 623

Ebisawa, K., Titarchuk, L. \& Chakrabarti, S. K., 1996, PASJ, 48, 1

Ebisawa, K. et al. 1994, PASJ 46, 375

Haardt, F., \& Marashi, L. 1993, ApJ 413, 507

Mitsuda, K. et al. 1984, PASJ 36, 741

Mitsuda, K. et al. 1989, PASJ 41, p. 97

Narayan, R. 1996, ApJ 462(1), 136-41

Shakura, N. I. \& Sunyaev, R. A. 1973, A\&A, 24, 337

Shapiro, S.L. \& Lightman, A.P. 1976 ApJ204, 555

Sunyaev, R.A., \& Titarchuk, L., 1980, A\&A 86, p. 121

Tanaka, Y., \& Lewin, W. H. G. 1995, in "X-ray Binaries", eds. W. H. G. Lewin, J. van Paradijs, \& E. P. J, van den Heuvel

Titarchuk, L. 1996, Integral Workshop, St. Malo, France

van der Klis, M. \& van Paradijs, J. 1994, A\&A 281(1), L17-20

van der Klis, M. 1994, ApJS 92, 511-519

Vargas, M. et al. 1996, ApJ in press

White, N.E. 1993, AIP Conf. Proc. 308, 53-60 
White, N.E., Swank, J.H., Holt, S.S. 1983, ApJ 270, 711

Zhang, S.N. et al. 1996a, A\&AS in press

Zhang, S.N. et al. 1996b, ApJ in press

Zhang, S.N. et al. 1997a, ApJ submitted

Zhang, S.N. et al. 1997b, ApJ in press

Zhang, S.N. et al. 1997c, ApJ submitted

\section{Discussion}

S. Chakrabarti: I am happy to see results of your recent observations which seem to require two mass accretion rates and show hard/soft transition with pivotal properties. The result is well in line with Chakrabarti \& Titarchuk (1995). 